Pacific Journal of Mathematics

GROUP REPRESENTATIONS AND THE ADAMS SPECTRAL J Ja es Mu ram 


\section{GROUP REPRESENTATIONS AND THE ADAMS SPECTRAL SEQUENCE}

\section{R. JAMES MILGRAM}

Homotopy groups admit primary operations analogous to the Steenrod operations in ordinary cohomology theory and a good understanding of them seems vital to interpreting patterns in the homotopy of spheres.

Also, it has been known for a long time that a type of Steenrod algebra acts in $\operatorname{Ext}_{A}\left(Z_{p}, Z_{p}\right)$ if $A$ is a cocommutative Hopf algebra. Recently, D. S. Kahn showed that in the $E_{2}$ term of the Adams spectral sequence $\operatorname{Ext}_{\mathscr{S}(2)}^{* *}\left(Z_{\iota}, Z_{2}\right)$, certain of these operations on infinite cycles converge to the graded elements associated to the actual homotopy operations. Also, on infinite cycles, he showed how this structure determined some differentials.

In this paper, we further explore the relations between the operations in $\operatorname{Ext}_{\mathscr{A}(p)}^{* *}\left(Z_{p}, Z_{p}\right)$ and differentials in the Adams spectral sequence. In particular, for elements which need not be infinite cycles, we prove

TheOREM 4.1.1. (a) There are operations $S q^{i}$ in $\operatorname{Ext}_{\Omega^{\prime}(2)}\left(Z_{2}\right.$, $Z_{2}$ ) so that

$$
\hat{o}_{2}\left(S q^{2}(a)\right)=\left\{\begin{array}{ll}
h_{0} S q^{i+1}(a), & i \equiv s(2) \\
0 & \text { otherwise }
\end{array},\right.
$$

for $a \in \operatorname{Ext}_{\stackrel{\leftrightarrow}{r}(2)}^{r, s}\left(Z_{2}, Z_{2}\right)$.

(b) There are operations $\mathscr{P}^{\imath}, \beta \mathscr{P}^{i}$ in $\operatorname{Ext}_{\mathscr{A}(p)}\left(Z_{p}, Z_{p}\right)$ for $p$ an odd prime so that

$$
\partial_{2}\left(\mathscr{P}^{i}(\boldsymbol{a})\right)=\alpha_{0} \beta \mathscr{P}^{i}(\boldsymbol{a}),
$$

for $a \in \operatorname{Ext}_{\mathscr{\sim}(p)}^{r, s}\left(Z_{p}, Z_{p}\right)$. (Here, $S q^{i}$ takes $\operatorname{Ext}^{s, r}$ homomorphicalIy to $\mathrm{Ext}^{s+i, 2 r}$ while $\mathscr{P}^{\imath}$ takes $\mathrm{Ext}^{s, r}$ to $\mathrm{Ext}^{s+(2 i-r)(p-1), p r}$, and $\beta \mathscr{P}^{i}\left(\mathrm{Ext}^{s, r}\right) \subset \mathrm{Ext}^{s+(2 i-r)(p-1)+1, p r}$.)

These operations are readily computable in the Ext groups. (Methods for calculating them are given in $\S 6$ and [2], [19].) For example, $S q^{0}\left(h_{i}\right)=h_{i+1}, S q^{1}\left(h_{i}\right)=h_{i}^{2}$ where $h_{i}$ is the nonzero element in $\mathrm{Ext}^{1,2^{i}}$ dual to $S q^{2^{i}}$. (Our notation for elements in Ext is that of [13].) Applying 4.1.1, $\partial_{2}\left(h_{i}\right)=h_{0} h_{i-1}^{2}$, which is nonzero for $i$ greater than 3. Consequently, we recover

CoRollary (Adams). An element of Hopf invariant one $(\bmod 2)$ exists in $\pi_{n+s}\left(S^{n}\right)$ if and only if $n>s$ and $s=1,3,7$.

(The Hopf invariant of a homotopy class $\alpha$ is nonzero in $Z_{p^{-}}$ cohomology if and only if $\alpha$ is represented by an infinite cycle in 
Ext $^{1}$.)

Similarly, the mod $p$ operations can be calculated. We have ([12])

$$
\mathscr{P}^{p^{i}(p-1)}\left(h_{i}\right)=h_{i+1},
$$

and $\alpha_{0} \beta \cdot \mathscr{P}^{p^{i}(p-1)}\left(h_{i}\right) \neq 0$ for $i \geqq 0$. Thus we recover the result of Liulevicius and Shimada on the elements of mod $p$ Hopf invariant one.

In $\S 6$, we calculate the complete action of the $S q^{i}$ operations in $\operatorname{Ext}_{\approx(2)}^{s, t}\left(Z_{2}, Z_{2}\right)$ for $t-s<42$. It turns out that routine relations among the various classes, together with the differentials of 4.1.1(a), determine all $\partial_{2}$ differentials in this range. (I am indebted to $\mathrm{M}$. Tangora for showing me how to obtain some of the more obscure $\partial_{2}$ differentials in this way.)

One surprising result is that $\partial_{2}\left(c_{2}\right)=h_{0} f_{1}$, a differential in the 41 stem which was overlooked in [13]. This differential, in turn, implies $\nu \Theta_{4} \neq 0$ (which contradicts a result in [13]) where $\Theta_{4}$ is the class corresponding to the Kervaire invariant 1 manifold in dimension 30 . This in turn forces a $\partial_{3}$ differential in the 34 stem. Once these two differentials are accounted for, there seem to be no further corrections necessary in [13], and we can thus assume the two primary components of $\pi_{t}^{s}\left(S^{0}\right)$ known for $t<44$. For further details, see [5].

Perhaps equally surprising, the technique used to prove Theorem 4.1.1 is purely geometric in nature. We never need mention secondary cohomology operations or even primary ones.

Next, we study some of the ways in which the operations imply higher order differentials. Here the answers are not as satisfactory as before. However, we do succeed in characterizing all primary differentials through $\partial_{20}$ on these elements. More exactly, Theorem 5.1.1 characterizes the first possible nonzero $\partial_{k}\left(S q^{i}(a)\right)$ for $k<20$, provided $\partial_{j}(a)=0$ for $j \leqq k+1$. However, there are no places in the first 40 stems where such differentials occur (except, indirectly, the differential $\left.\partial_{3}(r)=h_{1} d_{0}^{2}\right)$, so in the absence of examples, it seemed fruitless to pursue the matter further.

Also, there are further applications of these geometric techniques. For example, in $\S 7$ we give a very direct proof that $\Theta_{4}$ is nonzero. Moreover, by using similar techniques with the two-cell-complex $S^{15} \cup_{2} e^{16}$, together with the fact that $\left(\Theta_{3}\right)^{2}=(\sigma)^{4}=0$, one obtains a proof that $\Theta_{5}$ is nonzero. Similarly, using the result of Barratt-Mahowald that $\left(\Theta_{4}\right)^{2}=0$, one obtains the existence of $\Theta_{6}$ (see [22] for details).

Finally, I would like to take this opportunity to express my thanks to D. S. Kahn for sharing his insights with me, and the Centro de Investigation for their support while this research was carried out. 
1. Group representations and their Thom spaces.

1. We consider representations

$$
r: G \longrightarrow L
$$

where $G$ is a finite group and $L$ is the unitary group $U_{(n)}$ or the orthogonal group $O_{(n)}$. Any such representation gives rise to a map of classifying spaces

$$
B_{(r)}: B_{G} \longrightarrow B_{L}
$$

see e.g., [18]. By the Steenrod classification theorem $B_{(r)}$ is equivalent to specifying a principal $L$-bundle or its associated $n$-plane bundle over $B_{G}$. We denote this $n$-plane bundle by $B_{(r)}^{\sharp}\left(\xi_{n}\right)$ where $\xi_{n}$ is the universal bundle over $B_{L}$.

Definition 1.1. The representation $r$ is said to have $L$-filtration $k$ if the associated map $B_{(r)}$ is homotopically trivial on the $k-1$ skeleton of $B_{G}$.

This is equivalent, of course, to saying $B_{(r)}^{\sharp}\left(\xi_{n}\right)$ is trivial over the $k-1$ skeleton of $B_{G}$ and may also be rephrased as

Proposition 1.2. Let $E_{G}$ be the universal covering space of $B_{G}$; then the representation $r$ has filtration $k$ if and only if there is an r-equivariant map

$$
\Psi:\left(E_{G}\right)_{k-1} \longrightarrow L
$$

where $\left(E_{G}\right)_{k-1}$ is the $k-1$ skeleton of $E_{G}$ and r-equivariant means $\Psi(g x)=r(g) \Psi(x)$ for all $x \in\left(E_{G}\right)_{k-1}$.

Proof. Let $E_{L}$ be the universal principal $L$-bundle over $B_{L}$. Given a representation $r$ this induces an $r$-equivariant map

$$
\varphi: E_{G} \longrightarrow E_{L}
$$

moreover, by standard arguments (since $E_{L}$ is contractible and $E_{G}$ is $G$-free) any 2 such are equivariantly homotopic. Likewise, given any $r$-equivariant map $\Psi:\left(E_{G}\right)_{k-1} \rightarrow E_{L}$ it can be extended to an $r$-equivariant map $\varphi$. The induced map of classifying spaces $\bar{\varphi}$ will send the $k-1$ skeleton of $B_{G}$ to $* \in B_{L}$. To obtain the converse note that $B_{(r)}$ is covered by an equivariant map $E_{G} \rightarrow E_{L}$ and use the covering homotopy property.

2. By use of the Whitney sum maps

$$
\mu_{i j}: L_{i} \times L_{j} \longrightarrow L_{i+j}
$$


we can define Whitney sums of representations $\left(r \oplus r^{\prime}\right.$ is the composition

$$
\left.G \stackrel{\Delta}{\longrightarrow} G \times G \stackrel{r \times r^{\prime}}{\longrightarrow} L_{i} \times L_{j} \rightarrow L_{i+j}\right) .
$$

In particular, the positive integral multiples of $r$ are defined and we have

THeOREM 1.2.1. Let $L_{i}=U_{(i)}$ and suppose the order of $G$ is $s$. Suppose $r: G \rightarrow U_{(i)}$ has filtration $k<2 i$, then sr has filtration $k+2$.

Proof. The obstruction to compression of the $k$-skeleton is an element $\sigma$ in $H^{k}\left(B_{G}, \pi_{k}\left(B_{U}\right)\right)$. By naturality the obstruction to compressing $s r$ is $s \sigma$. However, $|G| \cdot H^{*}\left(B_{G}, Z\right)=0$ so $s \sigma=0$ and the theorem follows.

Similarly in the case $L_{i^{\prime}}=O_{i}$ we have

THEOREM 1.2.2. Let $m=(2|G|)^{2 s} 2^{\delta_{1}}|G|^{\delta_{2}}$ with $0 \leqq \delta_{2} \leqq \delta_{1}-1$, $0 \leqq \delta_{1} \leqq 2$ and let $r: G \rightarrow O_{n}$ be any representation, then $m r$ has filtration at least $8 s+2^{\delta_{1}}+4^{\delta_{2}}-1$ in $B_{0}$.

3. We now consider the Thom complex of the associated $n$-plane bundle $B_{(r)}^{\ddagger}\left(\xi_{n}\right)$. It admits a very simple description.

Definition 1.3.1. Let $B$ be a space with base point *, and $A$ be any space, then the half smash product

$$
A \ltimes B
$$

is $A \times B / A \times *$.

A representation $r: G \rightarrow L$ induces an action of $G$ on $S^{2^{k} n}(k=0$ if $L=0_{(n)}, k=1$ if $\left.L=U_{(n)}\right)$ induced from the action of $L$ on $S^{2^{k_{n}}}=$ $R^{2^{k} n} \cup \infty$. In terms of this action we have

THEOREM 1.3.2. The Thom complex of $B_{(r)}^{*}\left(\xi_{n}\right)$ is homeomorphic to

$$
E_{G} \ltimes{ }_{G} S^{2 k_{n}}
$$

Proof. The associated bundle $B_{(r)}^{*}\left(\xi_{n}\right)$ can also be described as

$$
E_{G} \times{ }_{G} R^{2_{n}}
$$

where the action of $G$ is that given above. Also, the associated sphere bundle admits a similar description. Hence, the theorem follows on equating the sphere bundle to the base point and using the fact that $L$ acts linearly in $R^{2^{k_{n}}}$. 
As an immediate corollary we have

THEOREM 1.3.3. If $r$ has filtration $p$ there is a G-equivariant map $\varphi:\left(E_{G}\right)_{p-1} \ltimes S^{2^{k_{n}}} \longrightarrow S^{2^{k_{n}}}$

which is of degree 1 on the bottom cell (i.e., $\varphi(x g, y)=\varphi(x, g y)$ ).

Proof. If $r$ has filtration $p$ then $B_{(r)}^{\sharp}\left(\xi_{n}\right)$ is trivial on the $p-1$ skeleton of $B_{G}$, so there is a bundle mapping

$$
\rho: B_{(r)}^{\sharp}\left(\xi_{n}\right) \mid\left(B_{G}\right)_{p-1} \longrightarrow R^{2^{k_{n}}}
$$

and an induced map Thom spaces $T(\rho):\left(E_{G}\right)_{p-1} \ltimes{ }_{G} S^{2^{k_{n}}} \rightarrow S^{2^{k_{n}}}$. Now $\phi$ is obtained by composing with the map

$$
\pi: E_{G} \ltimes S^{2^{k_{n}}} \longrightarrow E_{G} \ltimes S^{2^{k_{n}}} .
$$

4. In order to obtain alternate descriptions of these spaces we need to observe that two bundles will have homeomorphic Thom spaces if the bundles are isomorphic. In particular for representations $r_{1}, r_{2}$ this is equivalent to requiring that $B_{\left(r_{1}\right)} \simeq B_{\left(r_{2}\right)}$.

Proposition 1.4.1. $B_{\left(r_{1}\right)} \simeq B_{\left(r_{2}\right)}$ if there is an element $a$ in the identity component of $L$ so $r_{1}(g)=a^{-1} r_{2}(g) a$ for all $g \in G$.

Proof. Let $P$ be a path from the identity to $a$, then $P(t)^{-1} r_{2}(g) P(t)$ provides an equivariant homotopy from $r_{1}$ to $r_{2}$ and hence a homotopy of $B_{\left(r_{1}\right)}$ to $B_{\left(r_{2}\right)}$.

Since $U_{n}$ is connected we can apply 1.4.1 without difficulty and we find that the Thom complex of $B_{(r)}^{\sharp}\left(\xi_{n}\right)$ is homeomorphic to the Thom complex of a sum of irreducible representations which can usually be calculated.

Over $O_{n}$, however, we must be careful to check that the $a$ used to make $r_{1}$ equivalent to $r_{2}$ has determinant +1 and not -1 .

2. Some explicit representations and their Thom complexes.

1. Let $Z_{q}$ be a finite cyclic group with generator $T$ and let $R^{1}$ be the representation sending $T$ to $\rho=e^{2 \pi i / q}$. Then $R^{i}$ is the $i^{\text {th }}$ tensor product of $R^{1}$ with itself and sends $T$ to $\rho^{i}$. These $R^{i}$ form a complete set of irreducible representations of $Z_{q}$ and any unitary representation is uniquely equivalent to a sum of these.

Let $Z_{q}$ act on $S^{2 n-1}$ by $T\left(z_{1} \cdots z_{n}\right)=\left(\rho^{i_{1}} z_{1}, \cdots, \rho^{i_{n}} z_{n}\right)$.

The resulting quotient space is the Lens space

$$
L_{q}\left(i_{1}, \cdots, i_{n}\right) \text {. }
$$


We define a classifying space construction for $Z_{q}$ by letting $B_{Z_{q}}$ be the limit of the $L_{q}(1, \cdots, 1)$.

THEOREM 2.1.1. The Thom space of $m_{0}(I)+m_{1} R^{1}+\cdots+m_{q-1} R^{q-1}$ over $B_{Z_{q}}$ is homeomorphic to

$$
\lim _{s \rightarrow \infty} \Sigma^{m_{0}}\left(\frac{L_{m_{1}}^{L_{m_{1}} \underbrace{1 \cdots 1}_{m_{2}}} \cdots \underbrace{2 \cdots 2}_{m_{q-1}} \cdots \underbrace{q-1, \cdots q-1}_{m_{q-1}}), \underbrace{1 \cdots 1}_{s})}{L_{q}(\underbrace{1 \cdots 1}_{m_{1}} \cdots \underbrace{q-1, \cdots q-1})}\right)
$$

(Here $I$ is the trivial 1-dimensional representation.)

Proof. Embed $S^{2 k-1}$ in $S^{2 n-1}$ as the points $\left(0 \cdots 0, z_{1} \cdots z_{k}\right)$. Define an action of $Z_{q}$ on $S^{2 m-1}$ as $T\left(z_{1} \cdots z_{n-k}, \widehat{z}_{1} \cdots \widehat{z}_{k}\right)=\left(\rho^{i_{1}} z_{1} \cdots \rho^{i_{n-k}} z_{n-k}, \rho \widehat{z}_{1} \cdots\right.$ $\left.\rho \hat{z}_{k}\right)$. The embedding induces an inclusion $L_{q}(1 \cdots 1) \subset L_{q}\left(i_{1}, \cdots i_{n-k}\right.$, $1 \cdots 1$ ) with normal bundle $R^{i_{1}} \oplus \cdots \oplus R^{i_{n-k}}$. It is now evident that the Thom complex of $R^{i_{1}} \oplus \cdots \oplus R^{i_{n-k}}$ is $L_{q}\left(i_{1} \cdots i_{n-k} 1 \cdots 1\right) / L_{q} i_{1} \cdots$ $i_{n-k}$ ) since the normal bundle maps homeomorphically till it arrives at $L_{q}\left(i_{1} \cdots i_{n-k}\right)$, which is then the image of the sphere bundle.

For $Z_{2}$ and real representations we have 2 irreducible ones, the trivial one $I$ and the identity $R: Z_{2} \rightarrow Z_{2}=0(1)$, so we have

THEOREM 2.1.2. Let $R P^{\infty}$ be $B_{Z_{2}}$, then the Thom space of $m_{0} I+$ $m_{1} R$ is homeomorphic to

$$
\Sigma^{m_{0}}\left(R P^{\infty} / R P^{m_{1}-1}\right) .
$$

(The proof does not differ essentially from that of 2.1.1.)

2. We recall the characteristic classes of these representations.

Theorem 2.2.1. Let $\iota \in H^{2}\left(B_{Z_{q}}, Z\right)$ be the first Chern class of $R^{1}$, then the total Chern class of $R^{k}$ is $1+k \iota$. (This allows us to calculate, if necessary, the action of the Steenrod algebra $\mathscr{A}(p)$ in the Thom spaces above.)

3. Consider the representation $r_{n}: Z_{2} \rightarrow O_{2 n}$ defined by $r_{n}(T)(x, y)$ $=(y, x)$. From 1.3.2 the Thom complex of $r_{n}$ is $E_{Z_{2}} \aleph_{T} S^{n} \wedge S^{n}=$ $\lim _{m \rightarrow \infty} S^{m} \aleph_{T} S^{n} \wedge S^{n}$, where $T$ acts as the antipodal map on $S^{m}$ and as the interchange map on $S^{n} \wedge S^{n}$.

Proposition 2.3.1. $\quad r_{n}$ is equivalent to $n R \oplus n I$. Thus $\sum^{n} R P^{\infty} /$ $R P^{n-1}$ is homeomorphic to $E_{Z_{2}} \aleph_{T} S^{n} \wedge S^{n}$.

Proof. By changing coordinates we can assume $r_{n}(T)$ has the form 
$\left(\begin{array}{ccccc}M & 0 & 0 & 0 & 0 \\ 0 & M & 0 & \cdot & \cdot \\ \cdot & \cdot & M & \cdot & 0 \\ 0 & \cdot & \cdot & \cdot & M\end{array}\right)$ where $M$ is the matrix $\left(\begin{array}{cc}0 & 1 \\ 1 & 0\end{array}\right)$.

$\operatorname{But}\left(\begin{array}{ll}0 & 1 \\ 1 & 0\end{array}\right) \sim\left(\begin{array}{rr}1 & 0 \\ 0 & -1\end{array}\right)$ and 2.3 .1 follows.

Similarly we can consider the complex representation $r_{n}: Z_{q} \rightarrow$ $U_{q n}$ where $r(T)\left(x_{1} \cdots x_{q}\right)=\left(x_{q}, x_{1}, x_{2} \cdots x_{q-1}\right)$. Its Thom complex is $E_{z_{q}} \ltimes_{z_{q}} S^{n} \wedge \cdots \wedge S^{n}=\lim _{m \rightarrow \infty} S^{2 m-1} \aleph_{z_{q}} S^{n} \wedge \cdots S^{n}$ where $Z_{q}$ acts on $S^{2 m-1}$ by $T\left(z_{1} \cdots z_{m}\right)=\left(\rho z_{1}, \cdots, \rho z_{m}\right)$ and by the shift map in $S^{n} \wedge$ $\cdots \wedge S^{n}$.

Proposition 2.3.2. $\quad r_{n}$ is equivalent to $n\left[I \oplus R^{1} \oplus R^{2} \oplus \cdots \oplus R^{q-1}\right]$ so $E_{z_{p}} \ltimes_{z_{p}} S^{n} \wedge \cdots \wedge S^{n}$ is homeomorphic to

$$
\begin{aligned}
& \Sigma^{2 n} L_{p}(\underbrace{1 \cdots 1}_{n}, \cdots \underbrace{q-1, \cdots q-1}_{n}, 1,1 \cdots) / \\
& L_{p}(1, \cdots 1, \cdots q-1, \cdots q-1) \text {. }
\end{aligned}
$$

Proof. The characteristic polynomial of $r(T)$ is $\left(1-\lambda^{q}\right)^{n}$. Moreover, by changing coordinates $T$ becomes $\left(\begin{array}{cccc}M & 0 & \cdot & 0 \\ 0 & M & 0 & 0 \\ \cdot & \cdot & \cdot & \cdot \\ \cdot & \cdot & 0 & M\end{array}\right)$ where $M$ is the matrix $\left(\begin{array}{ccccc}0 & 1 & 0 & \cdot & \cdot \\ 0 & 0 & 1 & \cdot & \cdot \\ \dot{1} & \cdot & \cdot & \cdot & 1 \\ 1 & 0 & \cdot & \cdot & 0\end{array}\right)$. Thus, since $1-\lambda^{q}$ has $q$ distinct roots, $M$ can be diagonalized to $\left(\begin{array}{cccc}1 & 0 & \cdot & 0 \\ 0 & \rho & \cdot & 0 \\ \cdot & \cdot & \cdot & \cdot \\ 0 & \cdot & \cdot & \rho^{q-1}\end{array}\right)$ and 2.3 .1 follows.

4. As a final example we consider the symmetric group $\mathscr{S}_{4}$ and the representation $r_{n}: \mathscr{S}_{4} \rightarrow O_{4 n}$ defined by

$$
r(\alpha)\left(x_{1} \cdots x_{4}\right)=\left(x_{\alpha(1)} \cdots x_{\alpha(4)}\right) .
$$

The $Z_{2}$ cohomology of $B_{\mathcal{C}_{4}}$ is $P(A, B, C) / A C=0$ where $P(\quad)$ is a polynomial ring on three generators with $A$ of dimension $3, B$ of dimension 2 and $C$ of dimension 1 . Let $K$ be the Klein group. The inclusion $i: K \rightarrow \mathscr{S}_{4}$ induces the cohomology map $i:{ }^{*} H^{*}\left(\mathscr{S}_{4}\right) \rightarrow H^{*}(K)=$ $P\left(e_{1}, e_{2}\right)$ which sends $A \rightarrow e_{1}^{2} e_{2}+e_{1} e_{2}^{2}, B \rightarrow e_{1}^{2}+e_{1} e_{2}+e_{2}^{2}, C \rightarrow 0$. Next let $M$ be the group $Z_{2} \oplus Z_{2}$ and $j: Z_{2} \oplus Z_{2} \rightarrow \mathscr{S}_{4}$ be given by $j\left(T_{1}\right)=$ (12), $J\left(T_{2}\right)=(34)$. Then $j^{*}(A)=0, j^{*}(B)=e_{1} e_{2}, j^{*}(C)=e_{1}+e_{2}$

Theorem 2.4.1. The total Stiefel-Whitney class of $r_{n}$ is $(1+C+$ $B+A)^{n}$. 
Proof. Clearly $r_{n}$ is equivalent to $n r_{1}$. Hence we show the StiefelWhitney class of $r_{1}$ is $(1+C+B+A)$.

From the character tables we find

$$
\begin{aligned}
W\left(i^{*} r_{1}\right) & =\left(1+e_{1}\right)\left(1+e_{2}\right)\left(1+e_{1}+e_{2}\right) \\
& =1+i^{*}(B)+i^{*}(A) .
\end{aligned}
$$

Also $W\left(j^{*}\left(r_{1}\right)\right)=\left(1+e_{1}\right)\left(1+e_{2}\right)=1+j^{*}(C)+j^{*}(B)$. Now the result follows since

$$
i^{*} \oplus j^{*}: H^{*}\left(\mathscr{S}_{4}\right) \longrightarrow H^{*}(K) \oplus H^{*}\left(Z_{2} \oplus Z_{2}\right)
$$

is a monomorphism.

Thus, for example, the first 7 Stiefel-Whitney classes of $r_{2^{\prime}+7}, \iota>3$ are $C, B+C^{2}, A+C^{3}, C^{4}+C^{2} B, C^{5}+C B^{2}, B C^{4}+B^{3}+C^{6}+A^{2}, A B^{2}+$ $C^{7}$.

By generalizing slightly the techniques of [6] we can use these results to obtain a complete description of $H^{*}\left(\mathscr{S}_{n}, Z_{2}\right)$ for all $n$ (see e.g, $[20, \S 9])$ as well as the structure of the corresponding Thom complexes

$$
E_{S_{n}} \ltimes \mathscr{s}_{n} \underbrace{S^{m} \wedge \cdots \wedge S^{m}}_{n \text {-times }}
$$

We omit the details.

3. The geometry of the action of the Steenrod algebra on $\operatorname{Ext}_{\check{2}(p)}\left(Z_{p}, Z_{p}\right)$.

1. We start by recalling some well known facts from homological algebra. Throughout this paragraph we assume given a fixed augmented Hopf algebra $A$ over a ground ring $\Gamma\left(Z\right.$ or $Z_{p}$ for some prime $p$ ) and we require that the coproduct

$$
\Psi: A \longrightarrow A \otimes A
$$

be coassociative and cocommutative. We have

TheoRem 3.1.1. Suppose $\mathscr{C}$ is an A-free resolution of the augmentation, then for each subgroup $G$ of the symmetric group $\mathscr{S}_{n}$ there is a chain map

$$
\varphi_{G}: \mathscr{E}_{G} \otimes \mathscr{C} \longrightarrow \underbrace{\mathscr{C} \otimes \cdots \otimes \mathscr{C}}_{n \text {-times }}
$$

(where $\mathscr{E}_{G}$ is a $\Gamma(G)$ free resolution of the augmentation $\Gamma(G) \rightarrow \Gamma$ ), and

$$
\begin{aligned}
\varphi_{G}(\omega \otimes a c) & =\Psi^{(n)}(a) \varphi_{G}(\omega \otimes c) \\
\varphi_{G}(g \omega \otimes c) & =g \varphi_{G}(\omega \otimes c) .
\end{aligned}
$$

Moreover, given any other map $\varphi_{G}^{\prime}$ satisfying * there is an equivariant 
chain homotopy $H$ between $\varphi_{G}$ and $\varphi_{G}^{\prime}$ so that $H(\omega \otimes a c)=\Psi^{(n)}(a) H(\omega \otimes c)$.

Proof. (Compare [12]). Since $\Psi^{(n)}: A \rightarrow A^{n}$ is a map of algebras it induces a chain map

$$
\varphi_{0}: \mathscr{C} \longrightarrow \mathscr{C}^{(n)}
$$

and $\varphi_{0}(a c)=\Psi^{(n)}(a) \varphi_{0}(c)$. Now $*$ tells how to extend $\varphi_{0}$ over $\left(\mathscr{E}_{G}\right)_{0} \otimes$ $\mathscr{C}$. Note that since $\Psi$ is cocommutative and coassociative, $g \Psi^{n}=\Psi^{(n)}$ for each $g$ in $G$. Thus the various $g \varphi_{0}$ are all homotopic, and $\varphi_{G}(\alpha \times$ $\mathscr{C}$ ) is homotopic to 0 when regarded as a map $\varphi_{\alpha}: \mathscr{C} \rightarrow \mathscr{C}^{(n)}$ if $\alpha$ is in the kernel of the augmentation. Thus we can extend over the 1 skeleton of $\mathscr{E}_{G}$. The remaining arguments are equally direct.

Now, in the standard way (e.g. as in [25, Chapt. VII]) we can construct "cohomology operations" from the map $\bar{\phi}_{G}: \overline{\mathscr{E}}_{G} \otimes \overline{\mathscr{C}} \rightarrow \overline{\mathscr{C}}^{(n)}$ when $\overline{\mathscr{C}}$ is the reduced resolution of $A$. In particular this gives us operations coming from the cohomology of the symmetric group which have formal properties analogous to those of the ordinary Steenrod operations. Explicitly

THeOREM 3.1.2. Let $\Gamma=Z_{p}$ for $p$ an odd prime, then the $\varphi_{G}$ allow one to define operations $\mathscr{P}^{i}, \beta \mathscr{P}^{i}$ in $\operatorname{Ext}^{* *}{ }_{A}\left(Z_{p}, Z_{p}\right)$ with the following properties.

(i) $\mathscr{P}^{k}: \operatorname{Ext}^{i, j} \rightarrow \operatorname{Ext}^{i+(2 k-j)(p-1), p j}$

$\beta . \mathscr{P}^{k}: \mathrm{Ext}^{i, j} \rightarrow \mathrm{Ext}^{i+(2 k-j)(p-1)+1, p j}$

(ii) $\mathscr{P}^{j}, \beta \mathscr{P}^{j}$ are homomorphisms

(iii) Cartan formulae

$$
\begin{aligned}
\mathscr{P}^{i}(a b) & =\sum_{i \geq j \geq 0} \mathscr{P}^{j}(a) \mathscr{P}^{i-j}(b) \\
\beta \cdot \mathscr{P}^{i}(a b) & =\Sigma \beta \cdot \mathscr{P}^{j} a \cdot \mathscr{P}^{i-j} b+\Sigma(-1)^{(a)} \mathscr{P}^{j} a \beta \mathscr{P}^{j-j_{u}}
\end{aligned}
$$

(iv) Adem formulae

$$
\begin{aligned}
& \mathscr{P}^{a} \mathscr{P}^{b}=\sum_{t=0}^{[a / p]}(-1)^{a+t}\left(\begin{array}{c}
(p-1)(b-t)-1 \\
a-p t
\end{array}\right) \cdot \mathscr{P}^{a+b-t} \mathscr{P}^{t} \\
& \mathscr{P}^{a}\left(\beta \cdot \mathscr{P}^{b}\right)=\sum_{t=0}^{[a / p]}(-1)^{a+t}\left(\begin{array}{c}
(p-1)(b-t) \\
a-p t
\end{array}\right)\left(\beta \cdot \mathscr{P}^{a+b-t}\right) \mathscr{P}^{t} \\
& +\sum_{t=0}^{[(a-1) / p]}(-1)^{a+t+1}\left(\begin{array}{c}
(p-1)(b-t)-1 \\
a-p t-1
\end{array}\right) \mathscr{P}^{a+b-t}\left(\beta \cdot \mathscr{P}^{t}\right)
\end{aligned}
$$

(v) Normation $\mathscr{P}^{k}(a)=a^{p}$ if $a \in \mathrm{Ext}^{i, j}$, and $2 k=i+j$,

$$
\mathscr{P}^{k}(a)=0 \text { if } 2 k<j \text {. }
$$

TheoRem 3.1.3. For $p=2$ there are homomorphisms $S q^{i}$ : Ext** ${ }_{4}\left(Z_{2}, Z_{2}\right) \rightarrow \operatorname{Ext}^{* *}{ }_{4}\left(Z_{2}, Z_{2}\right)$ and

(i) $S q^{i}: \mathrm{Ext}^{k, j} \rightarrow \mathrm{Ext}^{i+k, 2_{j}}$ 
(ii) $S q^{i}(a b)=\sum S q^{j}(a) S q^{i-j}(b)$

(iii) $S q^{a} S q^{b}=\sum_{t=0}^{[a / 2]}\left(\begin{array}{c}b-t-1 \\ a-2 t\end{array}\right) S q^{a+b-t} S q^{t}$.

(The properties listed in 3.1.2, 3.1.3 follow directly from the corresponding properties in the homology of the symmetric groups $\mathscr{S}_{p}, \mathscr{S}_{p^{2}}$ and the proofs are in no way different from the corresponding proofs in [25] for the ordinary Steenrod operations. The mod $p$ case 3.1.2 appears in [12], however our grading of the mod 2 case differs from those to be found in [12], [2].)

Theorem 3.1.2 differs from the corresponding result for the ordinary Steenrod algebra in two ways. First, since there is no notion of a Bockstein homomorphism in $\operatorname{Ext}_{A}\left(Z_{p}, Z_{p}\right)$ if $A$ is a $Z_{p^{-}}$ module $\beta \mathscr{P}^{i}$ is not a Bockstein of $\mathscr{P}^{i}$, and, in fact is an entirely independent operation. Similarly, it is definitely not true that $\mathscr{P}^{0}$ is the identity operation (It is zero in general). Mod 2, $S q^{2 i+1}$ is independent of $S q^{2 i}$. Also note that $S q^{0}$ is neither zero nor the identity and both the $Z_{p}$ and $Z_{2}$ Adem relations preserve the number of terms, thus two-fold iterations $S q^{a} S q^{b}$ or $\mathscr{P}^{i} \mathscr{P}^{j}$ can only be equal to other two-fold iterations - a considerable simplication of the topological case. Finally we point out the important special example of the Adem relations,

$$
S q^{0} S q^{a}=S q^{a} S q^{0}
$$

2. Now our object is to imitate the construction of 3.1.1 geometrically. Thus, let

$$
S^{n} \supset Y_{n}^{1} \supset Y_{n}^{2} \supset \cdots \supset Y_{n}^{r} \supset \cdots
$$

be an Adams resolution of $S^{n}$ valid for a large range of dimensions and filtrations, and suppose

$$
S^{n p} \supset Z_{n p}^{1} \supset Z_{n p}^{2} \supset \cdots \supset Z_{n p}^{k} \subset \cdots
$$

is another such, then we have

THEOREM 3.2.1. Let $G \subset \mathscr{S}_{p}$ and suppose there is a G-equivariant $\operatorname{map}$

$$
u:\left(E_{G}\right)_{s} \ltimes \underbrace{S^{n} \wedge \cdots \wedge S^{n}}_{p \text {-times }} \rightarrow S^{n p}
$$

then $u$ is equivariantly homotopic to a map $\bar{u}$ so

$$
\bar{u}\left(E_{G}\right)_{t} \ltimes Y_{n}^{i_{1}} \wedge \cdots \wedge Y_{n}^{i_{p}} \subset Z_{n p}^{i_{1}+\cdots+i_{p}-t},
$$

moreover any two such $\bar{u}, \bar{u}^{\prime}$ are equivariantly homotopic via a homotopy $H$ with

$$
H\left\{I \times\left(E_{f}\right)_{t} \ltimes Y_{n}^{i_{1}} \wedge \cdots \wedge Y_{n}^{i_{p}}\right\} \subset Z_{n}^{i_{1}+\cdots+i_{p}-t-1} .
$$


(The proof proceeds by induction on the dimension of the skeleta of $E_{G}$, extending, first over a $G$-basis for the $i$-cells and then by $G$ equivariance. Similarly for the homotopy. This uses essentially the same analysis of the obstruction to compression as contained in 3.4 , 3.5 of [1].)

Thus $\bar{u}$ induces maps

3.2.2. $\left(\left(E_{G}\right)_{t},\left(E_{G}\right)_{t-1}\right) \ltimes_{G}\left(Y_{n}^{i_{1}}, Y_{n}^{i_{1}+1}\right) \wedge \cdots \wedge\left(Y_{n}^{i_{p}}, Y_{n}^{i_{p}+1}\right)$

$$
\longrightarrow\left(Z_{n p}^{i_{1}+\cdots+i_{p}-t}, Z_{n p}^{i_{1}+i_{2} \ldots+i_{p}-t+1}\right),
$$

which, since $Y^{i} / Y^{i+1}, Z^{j} / Z^{j+1}$ are just one point unions of $K\left(Z_{p}, n\right)$ 's through large ranges of dimensions, induces an $\mathscr{A}(p)$ map

$$
\mathscr{C} \longrightarrow \mathscr{E}_{G}^{*} \otimes{ }_{G} \mathscr{C}^{(n)} \text {. }
$$

This, in turn, defines a $G$-equivariant $\mathscr{A}(p)$ map

$$
\mathscr{E}_{G} \otimes \mathscr{C} \longrightarrow \mathscr{C}^{(n)},
$$

and thus is suitable for defining the action of the $\mathscr{P}^{i}$ in $\operatorname{Ext}_{\mathscr{N}(p)}\left(Z_{p}\right.$, $\left.Z_{p}\right)$. In particular, on the $E^{1}$ level the map $\bar{u}$ defines operations from 3.2.2 for each $t$-cell of $E_{G}$, which depend at the $E^{2}$ level only on the homology classes in $H_{*}(G)$ and the classes in $\operatorname{Ext}_{\mathscr{N}(p)}\left(Z_{p}, Z_{p}\right)$ involved. Thus, these are precisely the $\mathscr{P}^{i}$ operations, and their iterates.

3. Hence the existence of geometric models for the $\mathscr{P}^{i}, \beta \mathscr{P}^{i}, S q^{i}$ depends on the existence of appropriate maps

$$
\left(E_{G}\right)_{s} \aleph_{G} S^{n} \wedge \cdots \wedge S^{n} \longrightarrow S^{n q} .
$$

But these are given by 1.2.1, 1.2.2 and 1.3.3 if we start with the representations

$$
r^{q}: \mathscr{S}_{q} \longrightarrow O_{q},
$$

defined by $r^{q}(\alpha)\left(x_{1} \cdots x_{q}\right)=\left(x_{\alpha(1)}, \cdots, x_{\alpha(q)}\right)$.

We have

THEOREM 3.3.1. For a given $s$ there is an $n$ and an $\mathscr{S}_{q}$-map

$$
\varphi_{s}:\left(E_{S_{q}}\right)_{s} \ltimes \underbrace{S^{n} \wedge \cdots \wedge S^{n}}_{q \text {-times }} \longrightarrow S^{n} \wedge \cdots \wedge S^{n} .
$$

Moreover, let $G \subset \mathscr{S}_{q}$ and suppose there is an equivariant map

$$
\varphi:\left(E_{G}\right)_{s} \ltimes S^{n} \wedge \cdots \wedge S^{n} \longrightarrow S^{n} \wedge \cdots \wedge S^{n}
$$

then given any $G-\operatorname{map}$

$$
\omega: E_{G} \longrightarrow E_{\mathscr{S}_{q}}
$$


there is an $m$ so $\widetilde{\varphi}_{s}(\omega \times i d)$ is equivariantly homotopic to $\widetilde{\varphi}$ on $\left(E_{G}\right)_{s-1} \ltimes S^{n m} \wedge \cdots \wedge S^{n m}$.

(Here $\widetilde{\varphi}$ is defined as the composition

$$
\begin{aligned}
E_{\mho_{p}} \ltimes S^{n m} & \wedge \cdots \wedge S^{n m} \stackrel{(\Delta \times \text { shuff })}{\longrightarrow}\left(E_{\Im_{p}} \ltimes S^{n} \wedge \wedge S^{n}\right)^{(m)} \stackrel{\varphi^{(m)}}{\longrightarrow} S^{n m} \wedge \\
& \wedge \cdots \wedge S^{m n} \stackrel{\text { (shuff) }}{\longrightarrow} S^{n m} \wedge \cdots \wedge S^{n m}
\end{aligned}
$$

and similarly for $\widetilde{\varphi}$. The proof is entirely similar to the proof of 1.2.2 except applied to the obstruction to extending an equivariant homotopy.

3.3.1 shows that the operations obtained in this way are stably well defined. (For further details see [22, p. 205].)

4. The $\mathscr{P}^{i}$ operations and the $d_{2}$ differential in the Adams spectral sequence.

1. We pointed out in 3.1 that $S q^{2 i+1}$ is not the $Z_{2}$ Bockstein in any sense of $S q^{2 i}$; nor is $\beta \mathscr{P}^{i}$ the $Z_{p}$ Bockstein of $\mathscr{P}^{i}$, because there is no cohomological notion of $Z_{p}$ Bockstein in $\operatorname{Ext}_{\mathscr{A}(p)}\left(Z_{p}, Z_{p}\right)$. However, there is a homotopical notion of multication by $p$ which, in some sense, takes its place; namely, multiplication by $\alpha_{0}$ when $p$ is odd, and by $h_{0}$ for $p=2$. With this in mind, we state

THEOREM 4.1.1. Let $a \in \mathrm{Ext}^{r, s}{ }_{(p)}\left(Z_{p}, Z_{p}\right)$ (the $E_{2}$ term of the Adams spectral sequence); then

$$
d_{2} S q^{i}(a)= \begin{cases}h_{0} S q^{i+1}(\mathrm{a}), & i \equiv s(2) \\ 0 & \text { otherwise }\end{cases}
$$

for $p=2$.

$$
d_{2} \mathscr{P}^{i}(a)=\alpha_{0} \beta \mathscr{P}^{i}(a)
$$

for $p$ odd.

[Warning: The proof that follows appears to follow the formal algebra which would prove a similar result for a spectral sequence associated to a filtration of ordinary chain complexes. However, we are dealing with homotopy groups, so there is an error term, and the main work in the proof is to show that this error is in fact zero in the $E_{2}$ term of the Adams sequence, though it may definitely represent a nonzero element at level $E_{3}$ or $E_{4}$.]

Proof. (a) Consider the homotopy groups $\pi_{*}\left(Y^{i}, Y^{i+2}\right)$ for $S^{N} \supset$ $Y_{N}^{1} \supset \cdots \supset Y_{N}^{i} \supset \cdots$, a $Z_{2}$-Adams resolution of the sphere. There is an exact sequence

4.1.2 $\stackrel{\partial}{\longrightarrow} \pi_{j}\left(Y^{i+1}, Y^{i+2}\right) \stackrel{I}{\longrightarrow} \pi_{j}\left(Y^{i}, Y^{i+2}\right) \stackrel{J}{\longrightarrow} \pi_{j}\left(Y^{i}, Y^{i+1}\right) \stackrel{\partial}{\longrightarrow}$

where $\pi_{s}\left(Y^{k}, Y^{k+1}\right)$ is a $Z_{2}$ vector space isomorphic to $\bar{C}_{k, k+s}([1])$ for 
the associated resolution of $Z_{2}$ over $\mathscr{A}(2)^{1}$. (Of course, the $\partial$ map in 4.1.2 is just the $d_{1}$ differential.)

Hence $\pi_{j}\left(Y^{i}, Y^{i+2}\right)$ is a sum of $Z_{4}$ 's and $Z_{2}$ 's, and multiplication by $h_{0}$ corresponds to multiplication by 2 here. In particular if $J(B)$ represents $b$ in $E_{2}$ then the class $I^{-1}(2 B)$ which is well defined in $E_{2}$ represents $h_{0} b$.

Turning now to the case at hand suppose $a$ is represented by a specific map

$$
A:\left(D^{s-r}, S^{s-r-1}\right) \longrightarrow\left(Y^{r}, Y^{r+2}\right)
$$

and we are in the case $i \equiv s(2)$.

Thus if $v=\bar{u}(i d \times A \times A): E_{Z_{2}} \ltimes D^{r-s} \wedge D^{s-r} \rightarrow S$, we find that $v\left(e^{r-i} \ltimes\right.$ $\left.D^{s-r} \wedge D^{s-r}\right)$ represents $S q^{i}(a)$. Then

$$
\partial v\left(e^{r-i} \ltimes D^{s-r} \wedge D^{s-r}\right)=v\left[\left(T+(-1)^{r-i}\right) e^{r-i-1} \ltimes D^{s-r} \wedge D^{s-r}\right]
$$

$$
\begin{aligned}
& +v\left((-1)^{r-i} e^{r-i} \ltimes \partial D^{s-r} \wedge D^{s-r}\right) \\
& +v\left((-1)^{s-i} e^{r-i} \ltimes D^{s-r} \wedge \partial D^{s-r}\right) .
\end{aligned}
$$

(More precisely $\partial\left(e^{r-i} \ltimes D^{s-r} \wedge D^{s-r}\right)$ is the topological union of dises which we name in this way to stay as close as possible to the standard $\bigcup_{i}$ formulae for cochain operations [11].) The $\operatorname{disc}\left(e^{r-i} \ltimes \partial D^{s-r} \wedge\right.$ $\left.D^{s-r}\right)=M$ satisfies $v(M) \subset Y^{i+r+2}, v(\partial M) \subset Y^{i+r+3}$, and similarly $N=$ $\left(e^{r-i} \ltimes D^{s-r} \wedge \partial D^{s-r}\right)$ satisfies $v(N) \subset Y^{i+r+2},(\partial N) \subset Y^{i+r+3}$. Thus each of these discs represents a term $\{M\},\{N\}$ in $\pi_{*}\left(Y^{r+i+1}, Y^{r+i+3}\right)$, however $v\left(e^{r-i-1} \ltimes D^{s-r} \wedge D^{s-r}\right) \subset Y^{r+i+1}$ while part of its boundary, the two discs $e^{r-i-2} \ltimes D^{s-r} \wedge D^{s-r}, T e^{r-i-2} \ltimes D^{s-r} \wedge D^{s-r}$, is contained in $Y^{r+i+2}$, and similarly for $T e^{r-i-1} \ltimes D^{s-r} \wedge D^{s-r}$. Hence the formal decomposition of 4.1.3 is not quite valid in $\pi_{*}\left(Y^{i+r+1}, Y^{i+r+3}\right)$.

Now, from the Blakers-Massey theorem we can regard $\left\{v \partial e^{r-i} \ltimes\right.$ $\left.D^{s-r} \wedge D^{s-r}\right\}$ as contained in $\pi_{*}\left(Y^{i+r+1} / Y^{i+r+3}\right)$. Here the first two terms of 4.1.3 give a sphere $P$ as does each of $M, N$ and the boundary is $\{P\}+\{M\}+\{N\}$.

Also, note that $\left\{\partial v\left(e^{r-i+1} \ltimes \partial D^{s-r} \wedge D^{s-r}\right)\right\}=-\{M\}+\{N\}$, thus $\{P\}+\{M\}+\{N\} \sim\{P\}+2\{N\} \sim\{P\}$ since $\{N\} \in \operatorname{im}(I)$. It remains to prove $\{P\} \sim 2 \gamma$ where $\gamma$ satisfies $J(\gamma) \sim S q^{i+1}(a)$.

Since $v$ is equivariant there is a homeomorphism

$$
\begin{aligned}
h: e^{r-i-1} \ltimes D^{s-r} & \wedge D^{s-r} \rightarrow T e^{r-i-1} \ltimes D^{s-r} \wedge D^{s-r} \text { so } \\
v h=v . \quad \text { Since } & \partial e^{r-i-1} \ltimes D^{s-r} \wedge D^{s-r} \\
= & T e^{r-i-2} \ltimes D^{s-r} \wedge D^{s-r}-e^{r-i-2} \ltimes D^{s-r} \wedge D^{s-r}
\end{aligned}
$$

1 At this point, we indicate our stability conventions. We put

$$
\pi_{j}\left(Y^{k}, Y^{k+s}\right)=\lim _{N \rightarrow \infty} \pi_{N+j}\left(Y_{N}^{k}, Y_{N}^{k+s}\right) .
$$

Indeed, the absence of subscripts on the filtering spaces will always imply that we are considering stable groups. 
we have the homotopy factorization of $v$ on the boundary

$$
\partial(\quad) \stackrel{\pi}{\longrightarrow} S^{k} \vee S^{k} \stackrel{(1 \vee-1)}{\longrightarrow} S^{k} \stackrel{v}{\longrightarrow} S^{0} \text { where } k=2 s-r-i-2 .
$$

The iteration $(+1 \vee-1) \circ \pi$ is homotopically trivial. Let $I: Q \rightarrow S$ be such a homotopy. Now we can define a class $\gamma \in \pi_{*}\left(Y^{i+r+1} / Y^{i+r+3}\right)$ as $v(i d \times I)\left(e^{r-i-1} \ltimes D^{s-r} \wedge D^{s-r} \bigcup_{\partial} Q\right)$. Note that $J(\gamma) \sim S q^{i+1}(a)$. A homotopy class $\eta$ in $\pi_{k+1}\left(S^{k}\right)$ is defined as $I \bigcup_{k} I: Q \bigcup_{h \partial} Q \rightarrow S^{k}$ and we have easily

LEMMA 4.1.4. $\{P\}=2 \gamma+\eta\left\{v \cdot S^{k}\right\}$.

Finally, note that $\left\{v S^{k}\right\}$ is $(I)(\beta)$ for some $\beta$. Hence $\eta I(\beta)=$ $I(\eta \beta)$ and part a of 4.1.1 follows from

LEMMA 4.1.5. Let $S \supset Y^{1} \supset \cdots \supset Y^{i} \cdots$ be an Adams resolution (any prime $p$ ) and $a \in \pi_{*}\left(Y^{i}, Y^{i+1}\right)$ then for any element $\tau$ in $\pi_{i}(S)$ $i>0$, we have $\tau a=0$ in $\pi_{*}\left(Y^{i}, Y^{i+1}\right)$.

(Since in the Adams resolution $\tau$ has positive filtration the proof of multiplicativity in [1] shows $\tau \alpha$ is represented by an element of filtration at least $i+1$.)

The remaining case $i \not \equiv s(2)$ is analogous. However, here the homeomorphism $h$ is isotopic to the identity and the resulting error term is 0 . Part $b$ is similar to $a$. Again by standard arguments we arrive at $d_{2} \mathscr{P}^{i}(a)$ represented by $p \gamma+\tau\left\{S^{k}\right\}$ and 4.1.5 now gives the conclusion.

2. By the methods of [8] or [17], together with the results of 6.1 it is easy to prove

THEOREM 4.2.1 (a). $\operatorname{Ext}_{\varkappa_{p}}^{1, *}\left(Z_{p}, Z_{p}\right)$ is generated by elements $\alpha_{0}$, $h_{i} i \geqq 0, \mathscr{P}^{p^{i}(p-1)}\left(h_{i}\right)=h_{i+1}\left(h_{i}\right.$ is dual to $\left.\left|\mathscr{P}^{p^{i}}\right|\right)$ and $\alpha_{0} \beta \mathscr{P}^{p^{i}(p-1)}\left(h_{i}\right) \neq 0$ in $\operatorname{Ext}_{\approx p}^{3 *}\left(Z_{p}, Z_{p}\right)$.

(b) $\operatorname{Ext}_{\sim_{2}}^{1, *}\left(Z_{2}, Z_{2}\right)$ is generated by elements $h_{i} i \geqq 0\left(h_{i}\right.$ dual to $\left.S q^{2^{i}}\right)$ $S q^{0} h_{i}=h_{i+1}$, and $h_{0} h_{i}^{2} \neq 0$ in $\operatorname{Ext}_{{ }^{3}}{ }_{2}\left(Z_{2}, Z_{2}\right)$ for $i \geqq 3$.

(4.2.1 (a) was first proved in [12], and (b) is proved in [2].)

Thus $d_{2} h_{i}=\alpha_{0} \beta \mathscr{P}^{p i(p-1)}\left(h_{i}\right)$ for $i>0$ and $p$ odd, and the only elements which live to $E_{\infty}$ in $\operatorname{Ext}^{1}$ are $\alpha_{0}, h_{0}$. Similarly $d_{2}\left(h_{i}\right)=$ $h_{0} S q^{1} h_{i-1}=h_{0} h_{i-1}^{2}$ and the elements $h_{0}, h_{1}, h_{2}, h_{3}$ are the only survivors from $\operatorname{Ext}^{1}$ in $E_{\infty}$, for $p=2$.

Of course these differentials are well known [16] and it is also well known that their being nonzero is equivalent to the nonexistence of elements of Hopf invariant one in these dimensions, but 4.1.1 certainly provides the first purely geometric proof of these results.

3. It sometimes happens that there is a relation such as $h_{0} h_{2}^{2}=$ 0 which holds in $\operatorname{Ext}_{(2)}\left(Z_{2}, Z_{2}\right)$. The relation then "propagates". 
For example, let $\partial_{1} u=h_{0} h_{2}^{2}$, then $\partial_{1}\left(u \cup{ }_{1} u+h_{0} h_{2}^{2} \cup_{2} u\right)=h_{2}^{0} h_{3}^{2}, \partial_{1}\left(u \cup{ }_{2} u+\right.$ $\left.h_{0} h_{2}^{2} \cup_{3} u\right)=h_{1} h_{3}^{2}$. However, some of these cup- $i$ operations on $u$ ultimately become parts of classes in Ext, as with $\left(u^{2}+h_{0} h_{2}^{2} \cup_{1} u\right)=w$ since $\partial w=h_{1} h_{2}^{4}$ which equals 0 for another reason. When this occurs and we look more closely at this $\partial_{1}$ differential-say in $\pi_{*}\left(Y^{i}, Y^{i+2}\right)$ rather than $\pi_{*}\left(Y^{i}, Y^{i+1}\right)$ it turns out that the "filtration 2 " role of the differential is to make $\partial_{1}(v)=$ some element in higher filtration. In the example above, we have in fact

$$
\partial\left(u \cup_{1} u+h_{0} h_{2}^{2} \cup_{2} u\right)=h_{0}^{2} h_{3}^{2}+2(W+K)
$$

in $\pi_{*}\left(Y^{4}, Y^{6}\right)$ where $K=h_{1} V, \partial_{1} V=h_{2}^{4}$ say. This and relations similar to it sometimes account for higher differentials.

Consider, for example, the situation in dimensions 14 and 15

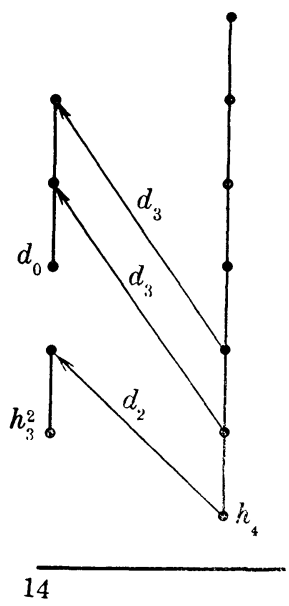

$d_{2}\left(h_{4}\right)=h_{0} h_{3}^{2}$ hence $d_{2}\left(h_{0} h_{4}\right)=h_{0}^{2} h_{3}^{2}$, but from the above remarks $h_{0}^{2} h_{3}^{2}=$ $h_{0} d_{0}$, hence $d_{2} h_{0} h_{4}=0$ but $d_{3} h_{0} h_{4}$ should equal $h_{0} d_{0}$. This would even be a proof if we had a better hold on $h_{0}^{2} h_{3}^{2}$ represented as a boundary above as compared with $h_{0}^{2} h_{3}^{2}$ represented as 4 times $h_{3}^{2}$. Such a program is possible and if carried out implies all known differential through the 45 stem in a more or less direct manner.

5. The $S q^{i}$ operation and higher differentials.

1. In [10] some theorems were proved on the connection of the $S q^{i}$ operations with $d_{3}, d_{9}$ under very restrictive hypothesis. Our object here is to remove most of these restrictions and increase the range of differentials considered. We have

THEOREM 5.1.1. Let $a \in \operatorname{Ext}^{i, t}\left(Z_{2)}\left(Z_{2}, Z_{2}\right)\right.$ and suppose $d_{s}(a)=0$ for $s<r$. Let $N=t-i+k$ for a given $k$ and define $\varphi(N)=8 m+2^{n}$ 
if $N+1=2^{4 m+n} c, c$ odd, $0 \leqq n<4$, then

$$
\begin{aligned}
& d_{\varphi(N)+1} S q_{k}(a)=h_{i} S q_{k-\varphi(N)}(a) \varphi(N)=2^{i}, i \leqq 3 \\
& d_{\varphi(N)+2} S q_{k}(a)=h_{1} h_{3} S q_{k-\varphi(N)}(a), \varphi(N)=9 \\
& d_{\varphi(N)+3} S q_{k}(a)=h_{1}^{2} h_{3} S q_{k-\varphi(N)}(a), \varphi(N)=10 \\
& d_{\varphi(N)+5} S q_{k}(a)=P^{1}\left(h_{2}\right) S q_{k-\varphi(N)}(a), \varphi(N)=12 \\
& d_{(N)+4} S q_{k}(a)=h_{0}^{3} h_{4} S q_{k-\varphi(N)}(a), \varphi(N)=16
\end{aligned}
$$

provided $\varphi(N)+q<r$ is satisfied in each case for the differential occurring.

In particular we mean to imply by this that all lower differentials on the specified elements are trivial. Also, note that $S q_{k}(a)=$ $S q^{i-k}(a)$. It is probable that the theorem can be strengthened so its conclusions hold even if $\varphi(N) \leqq r-q$. It is also likely that even for greater $\varphi(N)$ the differentials could still be obtained but now in terms of $d_{r}(a)$ as well.

2. To start the proof we "relativize" the construction of the $S q^{i}$ operations. Thus, suppose $a \in \pi_{j}\left(Y^{i}, Y^{i+2}\right)$ satisfies $d_{k}(a)=0 k<r$ then $a$ is the restriction of an element $\alpha$ in $\pi_{j}\left(Y^{i}, Y^{i+r}\right)$ and we can assume $a$ represented by a map $A:\left(D^{i}, S^{j-1}\right) \rightarrow\left(Y^{i}, Y^{i+r}\right)$. We have the diagram

5.2 .1



$\left(W_{Z_{2}}\right)_{t} \ltimes S^{j} \wedge S^{j} \quad \stackrel{i d \ltimes \hat{A} \wedge \hat{A}}{\longrightarrow} \quad W_{t} \ltimes Y^{i} / Y^{i+r} \wedge Y^{i} / Y^{i+r} \stackrel{\hat{u}}{\longrightarrow} S / Y^{2 i+r-t}$

where $\hat{i}:\left(D^{j}, S^{j-1}\right) \rightarrow\left(S^{j}, *\right)$ is the evident collapsing map.

The filtered map

5.2 .2

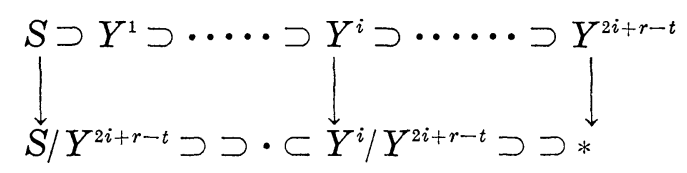

induces a map of spectral sequences which is clearly a monomorphism in all $E_{s}^{t}$ for $t<2 i+r-t$. Thus, if we can prove the existence of the stated differentials in the spectral sequence of the bottom row of 5.2.2 the theorem will follow

3. From the bottom row of 5.2.1 there is a map $\hat{u}: \sum^{j} P_{j}^{i+t} \longrightarrow S / Y^{2 i+r-t}$ so that $\sum^{j} e^{j+k} \subset Y^{2 i-k} / Y^{2 i+r-t}$.

We now define a "skeletal Adams filtration" of $\Sigma^{j} P_{i}^{j+t}$ so the map $\hat{u}$ 
becomes filtration preserving and thus induces a map of spectral sequences.

Lemma 5.3.1. Let $X$ be a finite $C W$ complex, then there is a spectral sequence converging to $\pi_{*}\left(X, Q_{2}\right)$ with

$$
\begin{aligned}
\widetilde{E}_{2} \cong \operatorname{Ext}_{\mathscr{N}(2)}\left(Z_{2}, Z_{2}\right) \otimes H_{*}\left(X, Z_{2}\right), \\
\quad\left(\widetilde{E}_{2}^{r, t}=\sum_{s} \operatorname{Ext}^{r+s, t}\left(Z_{2}, Z_{2}\right) \otimes H_{s}\left(X, Z_{2}\right)\right) .
\end{aligned}
$$

It is multiplicative in the sense that the pairing

$$
\operatorname{Ext}_{\mathscr{A}(2)}\left(Z_{2}, Z_{2}\right) \otimes \widetilde{E}_{2} \rightarrow \widetilde{E}_{2}
$$

commutes with differentials and induces pairings

$$
E_{r}\left(S^{0}\right) \otimes \widetilde{E}_{r}(X) \rightarrow \widetilde{E}_{r}(X)
$$

which also commute with differentials.

(Here $Q_{2}$ is the ring of fractions $a / b$ with $b$ prime to 2.) $)^{2}$

Proof. Suppose $X$ is $N$-dimensional. We start a resolution of $\Sigma^{k} X$ as the fiber in the evident map

$$
\varphi_{1}: \Sigma^{k} X \rightarrow K\left(H^{N}(X), N+k\right) \text {. }
$$

Let $F_{1}$ be the fiber of $\varphi_{1}$, then $F_{1}$ looks like the $N+k-1$ skeleton of $\Sigma^{k} X$ attached to $F_{1}^{\prime},{ }_{N+k}$ where $F_{1}^{\prime}$ is the fiber in the map similar to 5.3.2 for a boquet of spheres. Continue the resolution by killing all the cohomology which corresponds to $F_{1, N+k}^{\prime}$ and independently killing the cohomology from $H^{N-1}(X)$. The new fiber looks now like $\Sigma^{k} X_{N-2} \cup F_{1}^{\prime},{ }_{N+k-1} \cup F_{2}^{\prime},{ }_{N+k}$. Kill all the cohomology of $F_{2, N+k}^{\prime}$, independently that due to $F_{1, N+k-1}^{\prime}$, and that due to $H^{N-2}(X)$. The cohomology splitting again occurs so the process continues. (Note that this splitting is valid over $\mathscr{A}(2)$.) The resulting filtration of $\Sigma^{k}(X)$ gives the spectral sequence of 5.3.1 on taking the homotopy exact couple. We omit the remaining details as they are standard.

Corollary 5.3.3. Suppose $X$ is a finite K-connected $C W$ complex and there is a map

$$
f: X \rightarrow S^{0} / Y^{2 i+r-t}
$$

so that $f: X_{K+1} \subset Y^{2 i}, f: X_{K+2} \subset Y^{2 i-1}, \cdots, f: X_{K+j} \subset Y^{2 i-j+1}$ then $f$ induces a map of the spectral sequence 5.3.1 into the Adams sequence

${ }^{2}$ We must distinguish the spectral sequence introduced here from that developed in [15, Chapter III]. The bottom edge of Mahowald's sequence lies on the $s=0$ axis, while the bottom edge of ours lies on a line at a $-45^{\circ}$ angle to the $s=0$ axis. It is this change that allows us to compare our sequence with the Adams sequence. 
for $Y$ which is natural with respect to the action of the Adams sequence for a sphere on each.

(This follows directly from the proof of 5.3.1.)

4. In the situation of 5.3.3 we assume $X$ is $\Sigma^{j} P_{j}^{t+j}$ and the map $f: X \rightarrow S^{0} / Y$ is $\hat{u}$. Clearly $\widehat{u}_{\sharp}\left(\Sigma^{j} e_{j+s}\right)=S q^{i-s}(a)$ in the spectral sequence of 5.3.1. Thus it will suffice to prove the differentials of 5.1.1 occur in this spectral sequence. We now need

LEMma 5.4.1. For $L \geqq N-\varphi(N)$ we have

$$
\Sigma^{L} P_{N-\varphi(N)+1}^{N} \cong S^{N+L} \vee \Sigma^{L} P_{N-\varphi(N)+1}^{N-1} \cdot
$$

Moreover there is a map

$$
h: D^{N+L} \cup_{\alpha} S^{N+L-\varphi(N)} \longrightarrow \Sigma^{L} P_{N-\varphi(N)}^{N}
$$

so $h_{*}$ is a monomorphism in $\bmod 2$ homology and $\alpha$ is an odd multiple of the generator of $\operatorname{im}(J)$.

(Essentially 5.4.1 says the first nontrivial attaching map of any cell in a truncated projective space is $\operatorname{in} \operatorname{im}(J)$.)

Proof. $P_{N-\varphi(N)+1}^{N}$ is the Thom complex of $(N-\varphi(N)+1) \xi_{\varphi(N)-1}$, hence the normal bundle to $P^{\varphi(N)-1}$ is $\left(2^{q}-\varphi(N)\right) \xi$ but

so

$$
2^{q}-\varphi(N) \equiv N-\varphi(N)+1\left(\bmod 2^{q}\right)
$$

$$
P_{N-\varphi(N)+1}^{N}=\Sigma^{M}\left(P_{2 q-\varphi(N)}^{2 q-1}\right)
$$

and, since the top class of the Thom complex for the normal bundle is spherical the first statement follows.

To obtain the remaining statement note that by Atiyah's duality theorem (3.3 of [4]) the $S$-dual of $P_{N-\varphi(N)}^{N}$ is $P_{2^{q}}^{2^{q}+\varphi(N)}$. But this the Thom complex of $2^{q} \xi$ over $P^{\varphi(N)}$. Now, $2^{q} \xi$ is trivial over $P^{\varphi(N)-1}$ and it follows from [3] that using this trivialization there is a map

$$
P_{2^{q}}^{2^{q}+\varphi(N)} \longrightarrow S^{2^{q}} \mathbf{U}_{\alpha} e^{2^{\alpha}+\varphi(N)}
$$

where $\alpha$ is some odd multiple of the generator of (im $J$ ). Taking $S$-duals the result follows.

The proof of 5.1.1 follows since the $h_{i}, h_{1} h_{3}, P^{i}\left(h_{2}\right), h_{0}^{3} h_{4}$ are all known to carry the generator of $\operatorname{im}(J)$. Indeed this shows that in 5.3.1 the boundary of the relevant cell is the term desired plus an error term from lower cells. Checking the form of Ext in these early dimensions it is clear that the only possible term besides those in 5.1 .1 is

$$
\varepsilon\left(h_{3}^{2} S q_{k-\varphi(N)-3}(a)\right) \text { for all } \varphi(N)=12 .
$$

However, by checking secondary operations we can show this attach- 
ing map is 0 so $\varepsilon=0$ and the result follows.

5. It is easy to see how to generalize this to the $\bmod p$-case. In view of the results of $\S 2.3$ it would seem that the hardest step in doing this is to calculate sufficiently far into $\operatorname{Ext}_{\mathscr{A}(p)}\left(Z_{p}, Z_{p}\right)$ and still identify $\operatorname{im}(J)$.

REMARK 5.5.1. Recent results of Mahowald [14] enable one to completely identify $\operatorname{im}(J)$ in $\operatorname{Ext}_{\mathscr{N}(2)}\left(Z_{2}, Z_{2}\right)$. It seems reasonable to suppose the $\bmod (p)$ problem can be handled in a similiar way.

6. Calculation of the action of the Steenrod Algebra in $\operatorname{Ext}_{\sim_{p}}\left(Z_{p}, Z_{p}\right)$.

1. Some examples. We consider, mod $p$, the algebra $A=D P(\lambda)$ where $D P(\lambda)$ is a divided polynomial algebra. It is given a Hopf algebra structure by requiring that $\Delta\left(\lambda_{i}\right)=\Sigma \lambda_{j} \otimes \lambda_{i-j}$. Then for $p$ an odd prime

$$
\operatorname{Ext}_{A}^{* *}\left(Z_{p}, Z_{p}\right)=E\left(|\lambda|\left|\lambda_{p}\right|, \cdots,\left|\lambda_{p^{i}}\right|, \cdots\right) \otimes P\left(\theta_{1}, \theta_{p}, \cdots, \theta_{p^{i}} \cdots\right)
$$

where $\theta_{p^{i}}$ is dual to $\left|\lambda_{p^{i}}\right| \lambda_{p^{i}}{ }^{p-1} \mid$.

We calculate the $\mathscr{P}^{i}$ operations as

$$
\begin{aligned}
\text { THEOREM 6.1.1. } & \mathscr{P}^{p^{2}(p-1)}\left|\lambda_{p^{i}}\right|=c_{1}\left|\lambda_{p^{i+1}}\right| \\
& \beta \mathscr{P}^{p^{i}(p-1)}\left|\lambda_{p^{i}}\right|=c_{2} \theta_{p^{i}} \\
& \mathscr{P}^{p^{i}(p-1)+1}\left|\lambda_{p^{i}}\right|=0 \\
& \mathscr{P}^{(p-1) p^{i+1}+1} \theta=\left(\theta_{p^{i}}\right)^{p}
\end{aligned}
$$

where $c_{1}, c_{2}$ are nonzero constants, and this completely determines the action of $\tilde{\mathscr{A}}(p)$ here.

Proof. Consider in $\Delta^{(p)}\left|\lambda_{p^{i}}\right| \lambda_{p^{i}}{ }^{p-1} \mid$ the term $\left|\lambda_{p^{i}}\right| \otimes\left|\lambda_{p^{i}}\right| \otimes \lambda_{p^{i}} \otimes$ $\cdots \otimes \lambda_{p i}$. Clearly as we go through the successive constructions of the higher homotopies $M^{i}$ which give the $\mathscr{S}^{i}$ operations, we arrive at the term $\left|\lambda_{\rho^{i}}\right| \otimes \cdots \otimes\left|\lambda_{p^{i}}\right|$ in $M^{p-2}\left(\bar{\theta}_{p^{i}}\right)$. This proves the second statement, the first is virtually identical.

Similarly, for the same algebra $\bmod 2$ we have

$$
\operatorname{Ext}_{A}\left(Z_{2}, Z_{2}\right)=P\left(|\lambda|,\left|\lambda_{2}\right| \cdots\left|\lambda_{2^{i}}\right| \cdots\right)
$$

and for the $S q^{i}$ operations we find

$$
\begin{aligned}
\text { Theorem 6.1.2 } & S q^{1}\left|\lambda_{2^{i}}\right|=\left|\lambda_{2^{i}}\right|^{2} \\
S q^{0}\left|\lambda_{2^{i}}\right| & =\left|\lambda_{2^{i+1}}\right|
\end{aligned}
$$

and these determine the action of $\tilde{\mathscr{A}}(2)$ in $\operatorname{Ext}_{A}\left(Z_{2}, Z_{2}\right)$.

(The proof is unchanged from that of 6.1.1) 


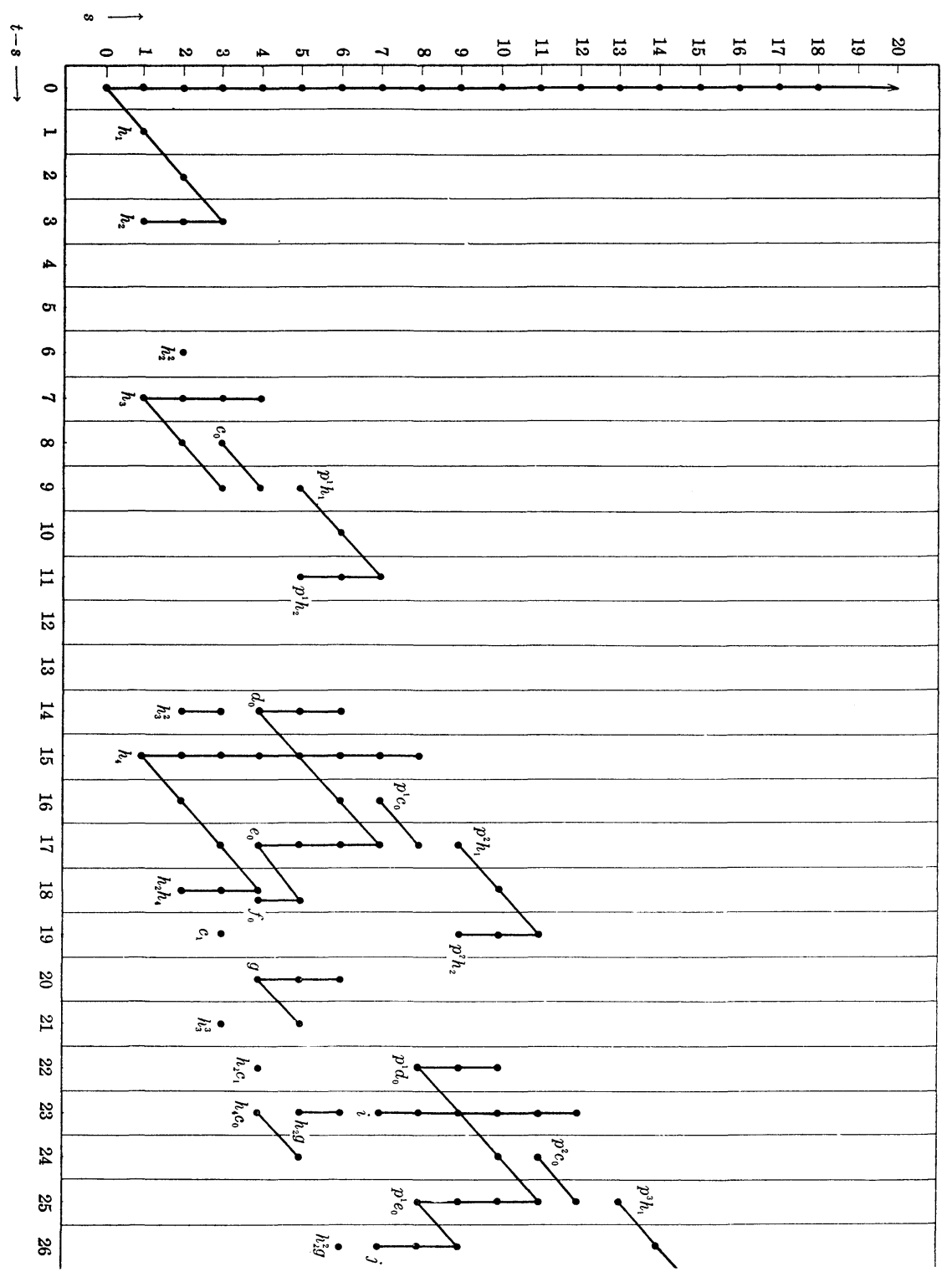

FIgURE $1\left(\begin{array}{cc}\text { Vertical lines denote multiplication by } h_{0} \\ 45^{\circ} & \text { lines denote multiplication by } h_{1}\end{array}\right)$

2. We now turn to the calculation of the $S q^{i}$ operations in $\operatorname{Ext}_{\mathscr{s}(2)}\left(Z_{2}, Z_{2}\right)$. Let $A^{2}$ be the sub-Hopf algebra of $\mathscr{A}(2)$ dual to the polynomial algebra $P\left(\xi_{1}\right)$ (using Milnor's notation see e.g., [21]). Then $\mathscr{A}(2) / / A^{2}=D P\left(\lambda_{1}\right)$ and consequently there is a map of algebras

$$
\omega: \operatorname{Ext}_{D P\left(\lambda_{1}\right)}\left(Z_{2}, Z_{2}\right) \longrightarrow \operatorname{Ext}_{\mathscr{N}(2)}\left(Z_{2}, Z_{2}\right)
$$



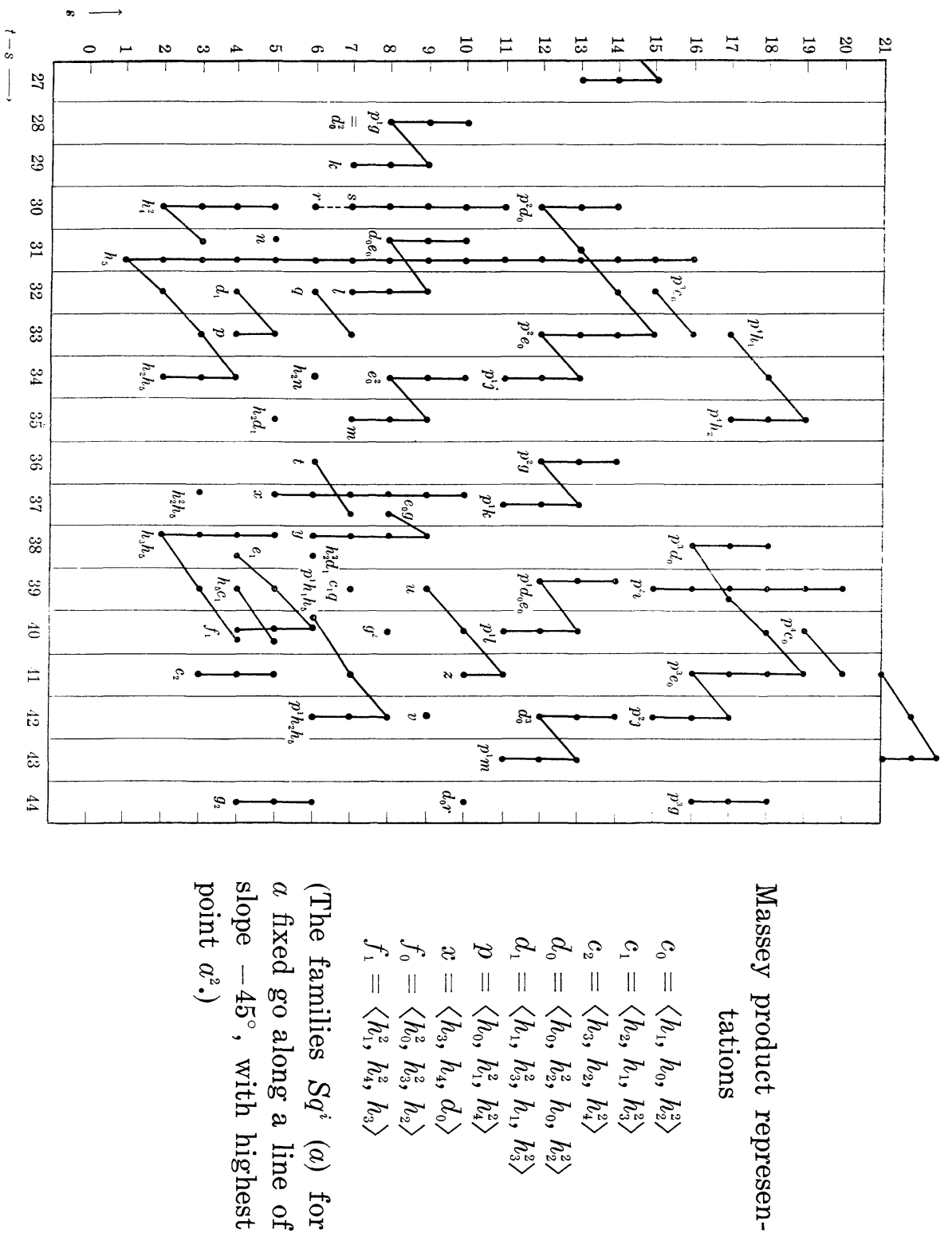

Figure 1 (Continued)

Clearly $\omega\left(\left|\lambda_{2^{i}}\right|\right)=h_{i}$ in $\operatorname{Ext}_{\mathscr{S}(2)}^{1,2^{i}}\left(Z_{2}, Z_{2}\right)$. Since $\omega$ is induced from a map of Hopf algebras $\omega\left(S q^{i}\right)=S q^{i}(\omega)$ and we can calculate the action of $\tilde{\mathscr{A}}(2)$ in the subalgebra of $\operatorname{Ext}_{\mathscr{A}(2)}\left(Z_{2}, Z_{2}\right)$ generated by the $\left(h_{i}\right)$.

The first generator which is not a polynomial in the $h_{i}$ is $c_{0}$ which has the Massey product representation $\left.<h_{1}, h_{0}, h_{2}^{2}\right\rangle$. (For further details and the "names" of various elements we refer to Figure 1.) 
Proposition 6.2.1. $S q^{3}\left(c_{0}\right)=c_{0}^{2}=h_{1}^{2} d_{0}$

$$
\begin{aligned}
& S q^{2}\left(c_{0}\right)=h_{0} e_{0} \\
& S q^{1}\left(c_{0}\right)=f_{0} \\
& S q^{0}\left(c_{0}\right)=c_{1}
\end{aligned}
$$

(The first statement and the fourth are known. The first following from [17], and the fourth from [19]. The second and third are shown using the techniques of [19] rather than the main theorem and explicitly writing representatives for these elements.) Thus, in view of 4.1.1 we find $d_{2}\left(f_{0}\right)=h_{0}^{2} e_{0}, d_{2}\left(h_{0} f_{0}\right)=d_{2}\left(h_{1} e_{0}\right)=h_{0}^{3} e_{0}=h_{1}^{3} d_{0}$ so $d_{2} e_{0}=$ $h_{1}^{2} d_{0}$, and it is only the differential $d_{3}\left(h_{0} h_{4}\right)=h_{0} d_{0}$ which is not due to 4.1.1, at least through the 21 stem.

3. Now we consider the algebra $A^{2}$ more closely. $\left(A^{2}\right)^{*}=$ $P\left(\xi_{2}, \xi_{3} \cdots \xi_{i} \cdots\right)$ with $\varphi\left(\xi_{i}\right)=\sum_{2 \leqq j \leq i-1} \xi_{j}^{2^{i-j}} \otimes \xi_{i-j}$. In particular $\left(\xi_{2}, \xi_{3}\right)$ generate a sub-Hopf algebra $B$ of $\left(A^{2}\right)^{*}$ and, if $A^{(4)}$ is the dual of $B$ in $A^{2}$, we have

$$
A^{2} / / A^{4}=D P\left(\lambda_{2}\right) \otimes D P\left(\lambda_{3}\right)
$$

Consequently there is a map

$$
u: \operatorname{Ext}_{D P\left(\lambda_{2}\right) \otimes D P\left(\lambda_{3}\right)}^{s, t}\left(Z_{2}, Z_{2}\right) \longrightarrow \operatorname{Ext}_{A^{2}}^{s, t}\left(Z_{2}, Z_{2}\right) .
$$

Proposition 6.3.1. $\operatorname{Ext}_{D P\left(\lambda_{2}\right) \otimes D P\left(\lambda_{3}\right)}\left(Z_{2}, Z_{2}\right)=\operatorname{Ext}_{D P\left(\lambda_{2}\right)} \otimes \operatorname{Ext}_{D P\left(\lambda_{3}\right)}$ as algebras over $\tilde{A}(2)$. Moreover for $t-s$ less than $50 u$ is onto and has kernel generated by

$$
\lambda_{2,2^{i}} \cdot \lambda_{2,2^{i+2}}, \lambda_{2,2^{2+3}} \cdot \lambda_{3,2^{i}}+\lambda_{3,2^{2}+2} \cdot \lambda_{2,2^{2}}, \lambda_{2,2^{2}}^{2} \cdot \lambda_{2,2^{3+i}}+\lambda_{22^{i+1}} \cdot \lambda_{2,2^{i+2}}^{2}
$$

and

$$
\lambda_{2,1}^{4} \cdot \lambda_{2,16}+\lambda_{2,4}^{5} \cdot
$$

Proof. The first part is evident. To prove the second we use the techniques of [8] and read off the differentials in the weighted augmentation filtration spectral sequence developed there.

This determines the action of $\mathscr{A} \widetilde{(2)}$ in $\operatorname{Ext}_{A^{2}}$ and in this range. Consider finally the map $v: A^{2} \rightarrow \mathscr{A}(2)$, this induces $v^{*}: \mathrm{Ext}_{s(2)} \rightarrow$ $\operatorname{Ext}_{A^{2}}$ and we have, again making use of [8]

$$
\text { Proposition 6.3.2. } \begin{aligned}
v^{*}\left(d_{0}\right) & =\left\{\left(\lambda_{22} \lambda_{22}\right)^{2}\right\} \\
v^{*}\left(e_{0}\right) & =\left\{\lambda_{21}\left(\lambda_{22}\right)^{3}\right\} \\
v^{*}(g) & =\left\{\lambda_{22}^{4}\right\} \\
v^{*} P^{1}\left(d_{0}\right) & =\left\{\lambda_{21}^{6} \lambda_{2}^{22}\right\} \\
v^{*} P^{1}\left(e_{0}\right) & =\left\{\lambda_{21}^{5} \lambda_{22}^{3}\right\} \\
v^{*}\left(d_{1}\right) & \left.=\left\{\lambda_{22} \lambda_{24}\right\}^{2}\right\} .
\end{aligned}
$$


$v^{*}(k)=\left\{\lambda_{21}^{2} \lambda_{22}^{5}\right\}, v^{*}(n)=\left\{\lambda_{21} \lambda_{22}^{4}\right\}$

4. From 6.3.2 we obtain $S q^{0}\left(d_{0}\right)=d_{1}$ (this is clear anyway from [19]) $S q^{0}\left(e_{0}\right)=e_{1}, S q^{2}\left(d_{0}\right)=r$ since $v^{*} S q^{2}\left(d_{0}\right)=\lambda_{22}^{6} \neq 0$ and the only element in $t-s=30$ and filtration 6 in $\operatorname{Ext}_{\mathscr{A}(2)}$ is $r$. Similarly $S q^{3}\left(e_{0}\right)=$ $m$ since $v^{*} S q^{3}\left(e_{0}\right)=\lambda_{22}^{7} \neq 0$ and $m$ is the only candidate. Also $S q^{2}\left(e_{0}\right)=$ $t$ since $S q^{2} v^{*}\left(e_{0}\right)=\lambda_{22}^{5} \lambda_{24} \neq 0$ and $t$ is the only candidate. Putting these together we have

THEOREM 6.4.1. $S q^{3}\left(d_{0}\right)=S q^{1}\left(d_{0}\right)=0$

$$
\begin{aligned}
& S q^{4}\left(d_{0}\right)=d_{0}^{2}, S q^{2}\left(d_{0}\right)=r, S q^{0}\left(d_{0}\right)=d_{1} \\
& S q^{4}\left(e_{0}\right)=e_{0}^{2}, S q^{3}\left(e_{0}\right)=m, S q^{2}\left(e_{0}\right)=t, S q^{1}\left(e_{0}\right)=x \\
& S q^{0}\left(e_{0}\right)=e_{1} .
\end{aligned}
$$

Proof. We first show $S q^{1}\left(e_{0}\right)=x$. From ([13, Proposition 5.1.3]) we have $h_{2} e_{0}^{2}=h_{0}^{4} x$. But from

$$
S q^{4}\left(h_{1} e_{0}\right)=h_{2} e_{0}^{2}=S q^{4}\left(h_{0} f_{0}\right)=h_{0}^{2} S q^{3}\left(f_{0}\right)
$$

it follows that

6.4.2.

$$
S q^{3}\left(f_{0}\right)=\varepsilon\left(h_{1} t\right)+h_{0}^{2} x
$$

Also

$$
S q^{3} f_{0}=S q^{3} S q^{1} c_{0}=S q^{2} S q^{2} c_{0}=S q^{2}\left(h_{0} e_{0}\right)
$$

by the Adém relation $S q^{2} S q^{2}=S q^{3} S q^{1}$. But

6.4.3. $S q^{2}\left(h_{0} e_{0}\right)=h_{0}^{2} S q^{1}\left(e_{0}\right)+h_{1} t$.

Now the result follows by comparing 6.4.2 with 6.4.3.

To verify $S q^{1}\left(d_{0}\right)=0$ note that the only candidate is $n$ but $v^{*}(n) \neq$ 0 while $S q^{1} v^{*}\left(d_{0}\right)=0$. Again, the only candidate for $S q^{3}\left(d_{0}\right)$ is $k$ but $v^{*}(k) \neq 0$ while $S q^{3}\left(v^{*}\left(d_{0}\right)\right)=0$. This completes the proof of 6.4.1.

CoRollary 6.4.4. $\quad d_{2} m=h_{0} e_{0}^{2}$.

COROLlaRY 6.4.5. $h_{0} r=s, h_{3} r=h_{1} t+h_{0}^{2} x$.

Proof. From 6.4.2, 6.4.3, 6.4.1 $S q^{2}\left(h_{2} d_{0}\right)=h_{3} r=h_{0}^{2} S q^{1}\left(e_{0}\right)+h_{1} t$. Now, since $h_{0} h_{3} r \neq 0$ it must follow that $h_{0} r=s$. (This result is asserted and an outline proof given in [13, 7.4], but the proof given here is much more direct.)

5. We now have

TheOREM 6.5.1. $S q^{0}\left(c_{1}\right)=c_{2}, S q^{1}\left(c_{1}\right)=f_{1}, S q^{2}\left(c_{1}\right)=h_{1} e_{1}, S q^{3} c_{1}=h_{2}^{2} d_{1}$, $S q^{0}\left(f_{0}\right)=f_{1}, S q^{1} f_{0}=0, S q^{2}\left(f_{0}\right)=y, S q^{3}\left(f_{0}\right)=h_{1} t+h_{0}^{2} x$. 
Proof. $S q^{0}\left(c_{1}\right)=c_{2}, S q^{0}\left(f_{0}\right)=f_{1}$ follow from [19], so $S q^{1}\left(c_{1}\right)=f_{1}$ since $S q^{0} S q^{1}=S q^{1} S q^{0}$. Now note that $h_{1} e_{0}^{2}=h_{0} e_{0} f_{0}=h_{0}^{2} m$. Thus $e_{0} f_{0}=$ $h_{0} m$ and $h_{2} m=h_{0}^{2} y \neq 0$ since $h_{2} e_{0} f_{0}=h_{1} e_{0} g$. Hence $S q^{3}\left(h_{0} f_{0}\right)=h_{0}^{2} S q^{2}\left(f_{0}\right)+$ $h_{1} S q^{3}\left(f_{0}\right)=h_{2} m+h_{1}^{2} t=h_{2} m$ since $h_{1}^{2} t=0$. We have previously shown $S q^{3}\left(f_{0}\right) . \quad S q^{1}\left(f_{0}\right)=S q^{1} S q^{1}\left(c_{0}\right)=0$ by the Adém relation. Similarly $S q^{0} S q^{2}\left(c_{0}\right)=h_{1} e_{1}=S q^{2}\left(S q^{0} c_{0}\right)=S q^{2}\left(c_{1}\right)$.

This essentially completes the description of the $S q^{i}$ operations in the range $t-s<45$. In particular we have

CoROLlaRy 6.5.2. $\quad d_{2}\left(c_{2}\right)=h_{0} f_{1}$

$$
d_{2}(y)=h_{0}^{3} x \text {. }
$$

This first differential was overlooked in [13] and to obtain the second differential they had to work considerably harder. The first differential also imples $h_{2} h_{4}^{2}$ is nonzero as a homotopy element [ $\nu \theta_{4} \neq$ 0] since $c_{2}$ has Massey product representation $\left\langle h_{3}, h_{2}, h_{4}^{2}\right\rangle$ which by [24] would converge to the Toda bracket $\left\{\sigma, \nu, \theta_{4}\right\}$ if this bracket were defined. This in turn can be shown to imply another differential overlooked in [13], namely $d_{3}\left(h_{2} h_{5}\right)=h_{0} p$. I should point out here that Barratt, Mahowald and Tangora independently discovered this error by investigating an apparent inconsistency in the 47 stem, and tracing it back [5]. The error was also found at about the same time by G.W. Whitehead using the semi-simplicial techniques of Kan-Curtis.

7. The use of the symmetric groups in homotopy theory.

1. So far the constructions $\mathrm{E}_{z_{p}} \ltimes X \wedge \cdots \wedge X$, respectively $E_{Z_{2}} \ltimes X \wedge X$, have been used by Toda [13], respectively Adams, Barratt and Mahowald, to obtain much information on homotopy groups. Basically they use the retractions supplied by $\S 3$ or other maps implied by the E.H.P. sequence. However it is clear from $\S \S 1$ and 2 that we can also use $E_{\mathscr{S}_{n}} \ltimes \underbrace{X \wedge \cdots \wedge X}_{n \text {-times }}$ to obtain information and it seems reasonable to assume that much remains to be done in this direction.

2. By way of an example we show how to use $\mathscr{S}_{4}$ to obtain a particularly simple proof that $\theta_{4}$ exists and is nonzero.

Consider $\sigma: S^{7} \rightarrow S^{0}$. This induces a map

$$
\widehat{u}(\sigma): E_{\mathscr{S}_{4}} \ltimes_{\mathscr{S}_{4}} S^{7} \wedge \cdots \wedge S^{7} \rightarrow E_{\mathscr{S}_{4}} \ltimes S^{0} \wedge \cdots \wedge S^{0} \stackrel{\hat{u}}{\longrightarrow} S^{0} .
$$

Now by the results of $\S 2.4$ we have $H^{*}\left(E_{\mathscr{S}_{4}} \aleph_{\mathscr{S}_{4}} S^{7} \wedge \cdots \wedge S^{7}\right)$ is $H^{*}\left(\mathscr{S}_{4}\right) \cup U$ and $S q^{1} U=C U, S q^{2} U=\left(B+C^{2}\right) U$. Consider now the map $Z_{2} \rightarrow \mathscr{S}_{4}$ by $T \rightarrow(13)(24)$. This induces a map $f: E_{\mathscr{S}_{2}} \ltimes_{\mathscr{S}_{2}} S^{14} \wedge S^{14}$ $\rightarrow E_{\mathscr{S}_{4}} \ltimes_{\mathscr{S}_{4}} S^{7} \wedge \cdots \wedge S^{7}$ and it is easy to check $f^{*}(B U)=e^{2} U^{\prime}$. Also 
there is a map $Z_{2} \oplus Z_{2} \rightarrow \mathscr{S}_{4}\left(T_{1} \rightarrow(12), T_{2} \rightarrow(34)\right)$ which induces a map

$$
g: E_{Z_{2} \oplus Z_{2}} \ltimes_{Z_{2} \oplus Z_{2}} S^{7} \wedge \cdots \wedge S^{7} \rightarrow E_{{ }_{4}} \ltimes S^{7} \wedge \cdots \wedge S^{7} \text {. }
$$

$g^{*}(C)=(e \otimes 1+1 \otimes e) U^{\prime} \quad g^{*}(B)=e \otimes e U^{\prime}$.

This last shows $C^{2}$ is dual to $\left(h_{3} \cup_{2} h_{3}\right) h_{3}^{2}$ while $B$ is dual to $h_{4}^{2}$. $h_{3} \cup_{2} h_{3}$ has filtration at least 2 so $\left(h_{3} \cup_{2} h_{2}\right) h_{3}^{2}$ has filtration 4 or more. Since $S q^{2} U=\left(B+C^{2}\right) U$ it follows that both these discs $h_{4}^{2},\left(h_{3} \cup_{2} h_{3}\right) h_{3}^{2}$ attach to $U$ by $\eta$. Hence their difference represents a sphere which must be called $h_{\ddagger}^{2}$ in the Adams spectral sequence.

\section{REFERENCES}

1. J. F. Adams, On the structure and applications of the Steenrod algebra, Comm. Math. Helv., 32 (1958), 180-214.

2. - On the non-existence of elements of Hopf invariant one, Ann. of Math. 72 (1960), 20-104.

3. - Vector fields on spheres, Ann. of Math. 75 (1962), 603-632.

4. M. F. Atiyah, Thom complexes, Proc. London Math. Soc., 11 (1961), 291-310.

5. M. Barratt, M. Mahowald and M. Tangora, Some differentials in the Adams spectral sequence II, Topology 9 (1970), 309-317.

6. H. Cardenas, El algebra de cohomologia del grupo simétrico de grado p2, Bol. Soc. Mat. Mexicana, 10 (1965), 1-30.

7. W. Feit, Characters of Finite Groups, Benjamin (1967)

8. V. K. A. M. Gugenheim and R. J. Milgram, On successive approximations in homological algebra, Trans. Amer. Math. Soc., 150 (1970), 157-182.

9. D. S. Kahn, A differential in the Adams spectral sequence, Proc. Amer. Math. Soc., 20 (1961), 188-191.

10. - Cup-i products and the Adams spectral sequence, Topology, 9 (1970), 1-10.

11. L. Kristensen, On the cohomology of two stage Postnikov systems, Acta Math., 107 (1962), 73-123.

12. A. Liulevicius, The factorization of cyclic reduced powers ky secondary cohomology operations, Mem. Amer. Math. Soc., 42 (1962).

13. M. Mahowald and M. Tangora, Some differentials in the Adams spectral sequence, Topology 6(1967), 349-369.

14. M. Mahowald, The order of the image of the J-homomorphism, Bull. Amer. Math. Soc., 76 (1970), 1310-1314.

15. - The metastable homotopy of $S^{n}$, Mem. Amer. Math. Soc., 72 (1967).

16. C. R. Maunder, Some differentials in the Adams spectral sequence, Proc. Cambridge Philos. Soc., 60(1964), 409-420.

17. J. P. May, Ph. D. thesis, Princeton University, 1964.

18. R. J. Milgram, The bar construction and abelian $H$-spaces, Illinois J. Math., 11 (1967), 242-250.

19. - Steenrod squares and higher Massey products, Bol. Soc. Mat. Mexicana, (1968), 32-51.

20. - The mod 2 spherical characteristic classes, Ann. of Math., 92 (1970), 238-261.

21. - A construction for s-parallelizable manifolds and primary homotopy operations, Topology of Manifolds, Markham (1970), 483-489.

22. - Symmetries and operations in homotopy theory, Amer. Math. Soc. Proc. Symposia Pure Math., 22 (1971), 203-211.

23. J. Milnor, The Steenrod algebra and its dual, Ann. of Math., 67 (1958), 150-171. 
24. M. Moss, Secondary compositions and the Adams spectral sequence, Topology, 10 (1971).

25. N. Steenrod and D. Epstein, Cohomology operations, Princeton University Press, Ann. of Math. Study 51 (1962).

26. H. TODA, Extended pth powers of complexes and applications homotopy theory, Proc. Japan Acad., 44(1968), 198-203.

Received October 19, 1970.

University of Illinois at Chicago Circle

AND

Stanford UNIVERSITy 


\section{PACIFIC JOURNAL OF MATHEMATICS}

\section{EDITORS}

H. SAMELSON

Stanford University

Stanford, California 94305

C. R. Hовву

University of Washington

Seattle, Washington 98105
J. DugundJI

Department of Mathematics

University of Southern California

Los Angeles, California 90007

RICHARD ARENS

University of California

Los Angeles, California 90024

\section{ASSOCIATE EDITORS}
E. F. BECKENBACH
B. H. NeumanN
F. WOLF
K. YOSHIDA

\section{SUPPORTING INSTITUTIONS}

UNIVERSITY OF BRITISH COLUMBIA

CALIFORNIA INSTITUTE OF TECHNOLOGY

UNIVERSITY OF CALIFORNIA

MONTANA STATE UNIVERSITY

UNIVERSITY OF NEVADA

NEW MEXICO STATE UNIVERSITY

OREGON STATE UNIVERSITY

UNIVERSITY OF OREGON

OSAKA UNIVERSITY
UNIVERSITY OF SOUTHERN CALIFORNIA

STANFORD UNIVERSITY

UNIVERSITY OF TOKYO

UNIVERSITY OF UTAH

WASHINGTON STATE UNIVERSITY

UNIVERSITY OF WASHINGTON

$\stackrel{*}{*} \stackrel{*}{*} \stackrel{*}{*}{ }^{*}$ AMERICAN MATHEMATICAL SOCIETY

NAVAL WEAPONS CENTER 


\section{Pacific Journal of Mathematics}

\section{Vol. 41, No. $1 \quad$ November, 1972}

Anatole Beck and Peter Warren, Weak orthogonality.................

Jonnie Bee Bednar and Howard E. Lacey, Concerning Banach spaces whose duals are abstract $L$-spaces.............................

Louis Harvey Blake, Canonical extensions of measures and the extension of regularity of conditional probabilities..........................

R. A. Brooks, Conditional expectations associated with stochastic processes ..........................................

Theodore Allen Burton and Ronald Calvin Grimmer, On the asymptotic behavior of solutions of $x^{\prime \prime}+a(t) f(x)=e(t) \ldots \ldots \ldots \ldots \ldots \ldots$

Stephen LaVern Campbell, Operator-valued inner functions analytic on the closed disc ............................................

Yuen-Kwok Chan, A constructive study of measure theory...

Alexander Munro Davie and Bernt Karsten Oksendal, Peak interpolation sets for some algebras of analytic functions ...................

H. P. Dikshit, Absolute total-effective $\left(N, p_{n}\right)(c, 1)$ method ...............

Robert E. Edwards, Edwin Hewitt and Kenneth Allen Ross, Lacunarity for

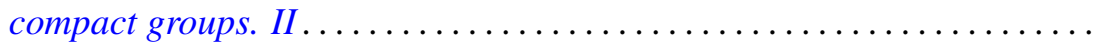

James Daniel Halpern, On a question of Tarski and a maximal theorem of Kurepa

Gerald L. Itzkowitz, A characterization of a class of uniform spaces that admit an invariant integral

Mo Tak Kiang, Semigroups with diminishing orbital diameters ..

Glenn Richard Luecke, A class of operators on Hilbert space ...

R. James Milgram, Group representations and the Adams spectral sequence. . .

G. S. Monk, On the endomorphism ring of an abelian p-group, and of a large subgroup...

Yasutoshi Nomura, Homology of a group extension ...

R. Michael Range, Approximation to bounded holomorphic functions on strictly pseudoconvex domains...

Norman R. Reilly, Inverse semigroups of partial transformations and $\theta$-classes.

Chris Rorres, Strong concentration of the spectra of self-adjoint operators

Saharon Shelah, A combinatorial problem; stability and order for models and theories in infinitary languages.

George Gustave Weill, Vector space decompositions and the abstract

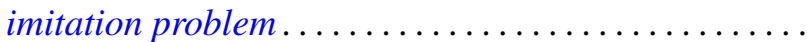

